\title{
Stability and symmetry of ion-induced surface patterning
}

\author{
Christopher S. R. Matthes $^{1 *}$ (D), Nasr M. Ghoniem ${ }^{1}$ and Daniel Walgraef ${ }^{2}$
}

\author{
*Correspondence: \\ csmatthes@gmail.com \\ 'University of California, Los \\ Angeles, 420 Westwood Plaza, Los \\ Angeles 90095, CA, USA \\ Full list of author information is \\ available at the end of the article
}

\begin{abstract}
We present a continuum model of ion-induced surface patterning. The model incorporates the atomic processes of sputtering, re-deposition and surface diffusion, and is shown to display the generic features of the damped Kuramoto-Sivashinsky (KS) equation of non-linear dynamics. Linear and non-linear stability analyses of the evolution equation give estimates of the emerging pattern wavelength and spatial symmetry. The analytical theory is confirmed by numerical simulations of the evolution equation with the Fast Fourier Transform method, where we show the influence of the incident ion angle, flux, and substrate surface temperature. It is shown that large local geometry variations resulting in quadratic non-linearities in the evolution equation dominate pattern selection and stability at long time scales.
\end{abstract}

\section{Introduction}

The erosion of surface material by ion sputtering is a fundamental process, which leads to the formation of surface roughness and patterns at the nanoscale. In some technological applications, sputtering erosion can be a significant factor in material degradation, while in others, nano-patterning by energetic particles can be a useful fabrication tool. The bombardment of solid surfaces with energetic ions initiates near surface collision cascades and the ejection of surface atoms. While a fraction of the ejected atoms may find their way back to be deposited on the surface, the majority travel farther away as the surface is eroded. The result of such atomistic events is a complex process of roughening, pattern formation, erosion and re-deposition; all of which have the ingredients of producing pattern-forming instabilities (Makeev et al. 2002).

Experimental evidence shows that ion sputtering can result in the formation of periodic surface patterns (Costantini et al. 2001; Habenicht 2001; Navez et al. 1962; Rusponi et al. 1997; Valbusa et al. 2002). The nature of these patterns, including their wavelength, amplitude, and orientation depends on many factors (e.g. ion energy, flux, angle of incidence, substrate temperature, and material properties). Considerable research has been performed by a number of groups to examine the effects of these many parameters on the surface features that develop under ion bombardment. The many experimental variables involved in the determination of the pattern spatial symmetry and periodicity make it imperative to develop a theoretical understanding that can guide experimental research. Notable existing theories of ion-induced patterning have been developed by Sigmund (1973), Bradley and Harper (1988), and Makeev et al. (2002).

(c) The Author(s), 2017 Open Access This article is distributed under the terms of the Creative Commons Attribution 4.0 International License (http://creativecommons.org/licenses/by/4.0/), which permits unrestricted use, distribution, and reproduction in any medium, provided you give appropriate credit to the original author(s) and the source, provide a link to the Creative Commons license, and indicate if changes were made. 
Among the key observations of ion-induced nano-patterning relate to the effects of the ion incidence angle, the ion fluence, and the surface temperature. It has been observed by Navez et al. (1962) that the bombardment of a clean glass surface with an ion beam produces surface morphology that is dependent on the ion beam incidence angle, $\theta$. The observed morphology was the formation of ripple structures oriented perpendicular to the ion beam for incidence angles close to normal $(\theta=0)$, and parallel to the ion beam for incidence angles close to grazing. Habenicht studied the effect of ion fluence on surface dynamics by exposing a highly ordered pyrolytic graphite (HOPG) surface with (0001) orientation increasing levels of ion fluence (Habenicht 2001). At high ion fluence the surface roughness increased as the nanostructures grew in size. Rusponi et al. (1997) studied the influence of temperature on the surface morphology of ion-bombarded silver. The results of their work showed that ion bombardment at low temperatures $(160 \mathrm{~K})$ resulted in a rough surface, but as the temperature was raised to $290 \mathrm{~K}$ and above, a ripple structure began to appear aligned along the $<1 \overline{1} 0>$ direction. The tendency of surface structures to orient along a particular direction is attributed to the enhanced surface diffusion that occurs at higher temperatures. On the surface of metals such as $\mathrm{Ag}(110)$ and $\mathrm{Cu}(110)$, the in-channel direction, $<1 \overline{1} 0>$ is an easy pathway for the diffusion of ad-atoms and vacancies compared with the cross-channel, or $<001>$ direction. Therefore, at higher temperatures where anisotropic surface diffusion is enhanced, the ripple structures have a quicker path to organize in that orientation (Costantini et al. 2001).

The early ideas of Sigmund provided the basis for Bradley and Harper to develop a continuum equation for surface evolution, from which the wavelength of the emerging pattern can be determined. More recently, Makeev et al. provided a comprehensive extension of the theory, where additional nonlinearities were incorporated as a result of a more rigorous analysis of the local geometry around the ion impact region. Nevertheless, the general framework remained consistent with earlier developments, with additional insights on casting the evolution equation into more familiar forms in the non-linear dynamics literature. In the present work, we extend these theoretical efforts further in two regards. First, we examine the stability and symmetry of evolving surface patterns with an analytical procedure. Second, we develop numerical solutions for the evolution of surface patterns that are consistent with the developed analytical method.

The main objective of the present work is to develop analytical and computational methods to further the understanding of surface pattern evolution under ion bombardment. We build on previous efforts by Sigmund (1973), Bradley and Harper (1988), and Makeev et al. (2002). Specifically, we aim at determination of the pattern wavelength and spatial symmetry at the later stages of surface evolution beyond the linear regime. Our efforts are confined to the main aspects of ion-induced surface instabilities, where the effects of surface stress, subsurface point defect generation, surface composition, impurities, mass redistribution, hydrodynamic effects, stochastic noise, and other contributing processes are excluded. We then develop a numerical method to describe the evolution of surface patterns, where the competition between erosion, redeposition, and surface diffusion is considered. We begin by reviewing the framework used to explain the underlying erosion mechanisms controlling surface morphology, as well as theoretical attempts to model deposition in "Background theory" section. Stability 
analysis of the governing equations is then explored in "Stability analysis" section, providing insight into the effects of nonlinearities on surface evolution and expected pattern formation. The numerical method for simulating surface evolution using an FFT algorithm is then put forward, and the results of the numerical simulations are presented in "Numerical simulations of pattern evolution" section. The interest here is to compare analytical theory to numerical simulations in order to reveal the role of nonlinear phenomena on pattern selection, stability, and long-term evolution. Lastly, a discussion of the numerical results of surface stability is given in "Numerical simulation results" section, and a summary of conclusions of the study is provided in "Conclusions" section .

\section{Background theory}

The continuum theory of surface erosion and stability due to energetic particle sputtering is reasonably well-established, and dates back to the work of Sigmund (1973). Several authors have added more features to the theory, and applied it to the understanding of surface nano-patterning and roughening. We will briefly introduce the theory here for completeness, while references (Makeev et al. 2002) and (Bradley and Harper 1988) provide more detailed descriptions.

When an obliquely-incident ion bombards the surface, it initiates a collision cascade downstream, leading to the removal of surface atoms that are energized by the Primary Knock-on Atom (PKA). Surface atoms that receive enough energy to break their bonds will be sputtered. If the surface location where the cascade initiates is concave (a local trough), more surface atoms will be closer to the PKA position than a convex surface, and thus more material will be removed. This fundamental idea was introduced by Bradley and Harper (1988), and it obviously leads to surface instabilities, since troughs will continue to be deeper as disproportionately more atoms are removed. Other phenomena can lead to modification of this behavior, for example, when surface diffusion is considered, it results in smoothing out of this geometry-dictated instability, and a periodic structure is obtained. Additional factors can also be introduced to account for surface stresses and point defect generation (Lauzeral et al. 1997; Walgraef et al. 1997), as well as higherorder non-linearities and noise (Makeev et al. 2002). Mass redistribution resulting from the momentum transfer by incident atoms has also been shown to be an influential factor in the dynamics of pattern formation (Muñoz-García et al. 2014). The presented study, however, is constrained to the primary known mechanisms for modeling ion sputtering, including curvature-induced erosion, temperature-induced surface diffusion, and the effect of nonlinearities and linear damping.

In Sigmund's theory of sputtering (Sigmund 1969,1973), the average energy deposited by an ion within the bulk of a material is assumed to be described by a Gaussian distribution, defined by the total energy deposited by the ion. The local surface geometry is expected to have a significant bearing on local material removal rate in Sigmund's model. Non-uniform profiles result in varying erosive effects, depending on the distance of a surface point from the center of the collision cascade, where an impinging ion releases its energy distribution. The analytical description of sputtering developed by Sigmund is important in developing a quantitative model for surface evolution. Using the approximation for the average energy deposition, Bradley and Harper (1988) showed that the yield depends on the curvature of the material surface. The following equation expresses the 
erosive velocity relative to the local normal vector of the surface (the $Z$-axes, see Fig. 1 for geometry definitions):

$$
v_{n}(\varphi, R)=\frac{J}{n} Y_{0}(\varphi)\left[\cos \varphi-\Gamma_{X}(\varphi) a / R_{X}-\Gamma_{Y}(\varphi) a / R_{Y}\right]
$$

where the coefficients $\Gamma_{X}$ and $\Gamma_{Y}$ are functions of the angle of incidence $\varphi$, as well as the collision cascade dimensions, $a$ and $\beta$. Here $a$ is the penetration depth of the ion along its trajectory, and $\beta$ is the energy distribution width, assuming isotropy in the $X$ and $Y$ directions (i.e. a spherical distribution) to greatly simplify the terms, as utilized by Valbusa et al. (2002). $Y_{0}(\varphi)$ represents the sputtering yield, and is defined by the ion energy, angle of incidence, and collision cascade dimensions. The atomic surface density is $n$, while the radii of curvature in the $X$ and $Y$ direction, $R_{X}$ and $R_{Y}$, respectively, are equivalent to the second derivatives of the local surface height, $Z$, in each direction.

Next, a transformation must be done between the local $(X, Y, Z)$ and laboratory coordinates $(x, y, h)$, as shown in Fig. 1 , to relate the normal velocity of erosion $v_{n}$ to the velocity of erosion along the $h$-axis (Cuerno and Barabási 1995), $\frac{\partial h}{\partial t}=-v_{n} \sqrt{g}$. Here $g \equiv 1+\left(\partial_{x} h\right)^{2}+\left(\partial_{y} h\right)^{2}$, so that $\sqrt{g}$ is the magnitude of the vector normal to the surface. The negative sign is needed to reflect the rate at which the hight decreases, as $v_{n}$ is simply the erosion velocity. The effect of surface diffusion on the rate of change of the surface height, $\mathrm{h}$, in the lab coordinate system has been analyzed by several authors (Asaro and Tiller 1972; Mullins 1957; Yang and Srolovitz 1994). It is included in the surface height evolution equation as:

$$
\frac{\partial h}{\partial t}=-v_{n} \sqrt{g}-K \nabla^{4} h,
$$

with $K=\frac{D_{s} \gamma \Omega^{2} \rho_{s}}{k T}$. Here $D_{s}$ is the surface diffusivity, $\gamma$ is the surface free energy, $\Omega$ is the atomic volume, $\rho_{s}$ is the atomic surface density, and $k$ is Boltzmann's constant, and $T$ is the substrate temperature. The first term of Eq. (2) may be expanded in an infinite series, and therefore approximations are needed to successfully model the expression. Bradley and Harper (BH) approximated the equation by expanding it to first order to produce a linear

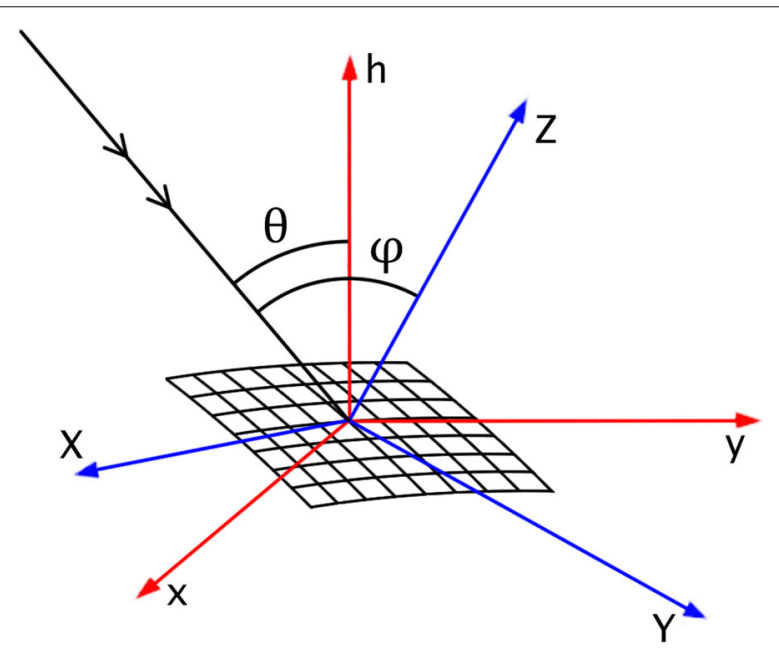

Fig. 1 Illustration of the local reference frame $(X, Y, Z)$ relative to the laboratory frame $(x, y, h) . \theta$ is the angle between the $h$-axis and the ion trajectory, and $\varphi$ is the angle between the local normal, $Z$, and the ion trajectory 
differential equation that describes the formation of surface ripples on an ion-bombarded surface (Bradley and Harper 1988):

$$
\frac{\partial h}{\partial t}=-v_{0}(\theta)+v_{0}^{\prime}(\theta) \frac{\partial h}{\partial x}-\frac{J a}{n} Y_{0}(\theta)\left[\Gamma_{X}(\theta)\left(\frac{\partial^{2} h}{\partial x^{2}}\right)+\Gamma_{Y}(\theta)\left(\frac{\partial^{2} h}{\partial y^{2}}\right)\right]-K \nabla^{4} h .
$$

This linear BH equation is a useful model for predicting ripple formation and explaining experimentally observed processes, such as ripple orientation and the flux and ion energy dependence of the wavelength (Makeev et al. 2002). However, the equation is limited to small local changes in the surface curvature, where differences between local and global (lab) frames are small.

Makeev et al. (2002) sought to address these limitations of the BH equation, accounting for processes such as lateral growth and kinetic roughening. Expansion of the first term in Eq. (2) that connects local-to-global coordinates was approximated to fourth order to yield:

$$
\begin{aligned}
\frac{\partial h}{\partial t}= & -v_{0}+\gamma \frac{\partial h}{\partial x}+\xi_{x}\left(\frac{\partial h}{\partial x}\right)\left(\frac{\partial^{2} h}{\partial x^{2}}\right)+\xi_{y}\left(\frac{\partial h}{\partial y}\right)\left(\frac{\partial^{2} h}{\partial y^{2}}\right)+\hat{v}_{x} \frac{\partial^{2} h}{\partial x^{2}}+\hat{v}_{y} \frac{\partial^{2} h}{\partial y^{2}} \\
& +\Omega_{1} \frac{\partial^{3} h}{\partial x^{3}}+\Omega_{2} \frac{\partial^{3} h}{\partial x \partial y^{2}}-D_{x y} \frac{\partial^{4} h}{\partial x^{2} \partial y^{2}}-D_{x x} \frac{\partial^{4} h}{\partial x^{4}}-D_{y y} \frac{\partial^{4} h}{\partial y^{4}}-K \nabla^{4} h \\
& +\frac{\lambda_{x}}{2}\left(\frac{\partial h}{\partial x}\right)^{2}+\frac{\lambda_{y}}{2}\left(\frac{\partial h}{\partial y}\right)^{2}+\eta(x, y, t),
\end{aligned}
$$

where the noise $\eta(x, y, t)$ represents random fluctuations in the evolution, and the coefficients are defined by the collision cascade parameters and angle of ion incidence, as defined by Makeev et al. (2002). Equation 4 is a nonlinear partial differential equation that is similar to generic forms in the non-linear dynamics literature. For example, the Kardar-Parisi-Zhang (KPZ) equation (Edwards and Wilkinson 1982; Godrèche 1991) was used to describe crystal growth by atomic beams at different length scales (Villain 1991). On the other hand, the Kuramoto-Sivashinsky (KS) equation is a deterministic, nonlinear equation originally developed to describe chemical turbulence (Kuramoto and Tsuzuki 1976) and laminar flame fronts (Sivashinsky 1977). The KS equation has been shown to offer a similar analytical description of the surface as the KPZ equation (Makeev et al. 2002). In addition, a damping term may be added to produce the Damped KuramotoSivashinsky (DKS) equation.

$$
\frac{\partial h}{\partial t}=-\alpha h-|v| \nabla^{2} h-K \nabla^{4} h+\frac{\lambda}{2}(\nabla h)^{2}
$$

The damping term results in smoothing of all spatial frequencies, thereby inhibiting kinetic roughening (Keller and Facsko 2010). Its presence in the continuum equation for ion-bombarded surfaces has been used to account for redeposition of sputtered material (Facsko et al. 2004; Keller and Facsko 2010), although this has been disputed (Bradley 2011).

At this point, solutions to Eq. (2) have to rely on approximations to the order of non-linearities resulting from the expansion of $\sqrt{g}$. Although Eq. (4) was introduced by Makeev et al., it is an approximation to an infinite series expansion, and contains nonlinearities beyond the original $\mathrm{BH}$ equation. Following the theory outlined by the original BH model (Bradley and Harper 1988) and following Makeev et al's analysis (Makeev et al. 2002), we further approximate the height evolution equation in a way that is consistent with elements of the KPZ and KS equation forms. Neglecting non-linearities associated 
with higher order gradients (third and fourth terms in Eq. (4)), and approximating the expansion to the lowest order nonlinearity, we finally use the form:

$$
\begin{aligned}
\frac{\partial h}{\partial t}=-\alpha h-v_{0} & +\frac{F a}{2}\left[\hat{v}_{x}\left(\frac{\partial^{2} h}{\partial x^{2}}\right)+\hat{v}_{y}\left(\frac{\partial^{2} h}{\partial y^{2}}\right)\right] \\
& +\frac{F a_{\beta}^{2}}{2}\left[\hat{v}_{x}\left(\frac{\partial h}{\partial x}\right)^{2}+\hat{v}_{y}\left(\frac{\partial h}{\partial y}\right)^{2}\right]-K \nabla^{4} h
\end{aligned}
$$

where the coefficients are defined as: $\hat{v}_{x}=2 s^{2}-c^{2}-a_{\beta}^{2} s^{2} c^{2} \quad \hat{v}_{y}=-c^{2}, \quad \hat{v}_{x}=3 s^{2} c-$ $c^{3}-a_{\beta}^{2} s^{2} c^{3}, \quad \hat{v}_{y}=-c^{3}$, with $c=\cos \varphi$ and $s=\sin \varphi$. In this expression, the following is defined as $F=\frac{J \varepsilon p a_{\beta}^{2} e^{-a_{\beta}^{2} / 2}}{2(2 \pi)^{1 / 2}}$, where $J$ is the ion flux, $\varepsilon$ is the ion energy, and $a_{\beta}=a / \beta$ characterizes the collision cascade size. Equation (6) is the time evolution equation of the surface in the laboratory frame. This expression has been simplified for normal ion incidence $(\theta=0)$, and by assuming an isotropic, or spherical energy distribution for the collision cascade (i.e. a radius of $\beta$ ).

The damping term depends directly on the surface position, $h$, rather than its derivatives, indicating that its effect goes beyond the scope of Sigmund's theory of sputtering (Sigmund 1973). The influence of this term on the surface morphology may be explained as the self-deposition of sputtered material resulting from ion bombardment (Facsko et al. 2004). When patterning is present, a significant amount of material may be deposited due to line-of-sight interaction of the sputtered material with adjacent surface features. This effect is more significant in the surface troughs rather than the peaks, thereby producing a damping effect to the curvature instabilities described in the Bradley-Harper model (Bradley and Harper 1988).

\section{Stability analysis}

\section{Linear stability analysis}

Linear stability of the evolution equation is examined by submitting the system to small perturbations and observing their temporal evolution. For small perturbations, the nonlinear terms of the governing equation may be neglected. Performing a Galilean transformation and excluding nonlinear terms, a non dimensionalized form of Eq. (6) may be written as:

$$
\frac{\partial \bar{h}}{\partial \tau}=-\bar{\alpha} \bar{h}+\hat{v}_{x}\left(\frac{\partial^{2} \bar{h}}{\partial X^{2}}\right)+\hat{v}_{y}\left(\frac{\partial^{2} \bar{h}}{\partial Y^{2}}\right)-\bar{K}\left(\frac{\partial^{4} \bar{h}}{\partial X^{4}}+2 \frac{\partial^{4} \bar{h}}{\partial X^{2} \partial Y^{2}}+\frac{\partial^{4} \bar{h}}{\partial Y^{4}}\right) .
$$

where $\bar{h}=\frac{a_{\beta}^{2}}{a} h, X=\frac{2 a_{\beta}}{a} x, \tau=\frac{2 F a_{\beta}^{2}}{a} t, \bar{\alpha}=\frac{a}{2 F a_{\beta}^{2}} \alpha, \bar{K}=\frac{8 a_{\beta}^{2}}{F a^{3}} K$. Performing a Fourier transform on Eq. (7), it may be written in Fourier space as:

$$
\sigma_{\tau} \bar{h}=\left[-\bar{\alpha}-\hat{v}_{x} q_{x}^{2}-\hat{v}_{y} q_{y}^{2}-\bar{K}\left(q_{x}^{2}+q_{y}^{2}\right)^{2}\right] \bar{h} .
$$

It has been shown that $\hat{v}_{x}$ and $\hat{v}_{y}$ are negative for a wide range of incidence angles (Bradley and Harper 1988) so that the linear growth rate of perturbations may become positive inducing the instability of uniform profiles. Effectively, considering the magnitude of the coefficients only, and dividing out $\bar{h}$ leads to the following growth rate for the amplitude of the spatial mode $\vec{q}$ where $q^{2}=q_{x}^{2}+q_{y}^{2}$.

$$
\sigma_{\tau}=-\bar{\alpha}+\left|\hat{v}_{x}\right| q_{x}^{2}+\left|\hat{v}_{y}\right| q_{y}^{2}-\bar{K}\left(q_{x}^{2}+q_{y}^{2}\right)^{2}
$$


For $\left|\hat{v}_{x}\right|>\left|\hat{v}_{y}\right|$, the maximum growth rate corresponds to a critical wavenumber defined as $q_{c}^{2}=\frac{\left|\hat{v}_{x}\right|}{2 \bar{K}}$, allowing us to rewrite the growth rate into the usual expression for anisotropic systems close to a pattern forming instability:

$$
\begin{aligned}
\sigma_{\tau} & =\left(-\bar{\alpha}+\left|\hat{v}_{x}\right| q^{2}-\bar{K} q^{4}\right)-\left(\left|\hat{v}_{x}\right|-\left|\hat{v}_{y}\right|\right) q_{y}^{2} \\
& =\epsilon-\bar{K}\left(q^{2}-q_{c}^{2}\right)^{2}-\left(\left|\hat{v}_{x}\right|-\left|\hat{v}_{y}\right|\right) q_{y}^{2}
\end{aligned}
$$

where $\epsilon=\frac{\hat{v}_{x}^{2}}{4 \bar{K}}-\bar{\alpha}$. For positive values of $\epsilon$, where $\bar{\alpha}$ is less than $\frac{\hat{v}_{x}^{2}}{4 \bar{K}}$, spatial modes with $\vec{q}= \pm q_{c} \overrightarrow{1}_{x}$ are unstable, that is $\vec{q}= \pm \sqrt{\frac{\left|\hat{\mid}_{x}\right|}{2 \bar{K}}} \overrightarrow{1}_{x}$.

On varying the beam incidence angle, the difference between $\left|\hat{v}_{x}\right|$ and $\left|\hat{v}_{y}\right|$ varies, and $\left|\hat{v}_{y}\right|$ may become greater than $\left|\hat{v}_{x}\right|$. In this case, the maximum linear growth rate corresponds to the modes with $\vec{q}= \pm \sqrt{\frac{\hat{v}_{y}}{2 \bar{K}}} \overrightarrow{1}_{y}$ and instability occurs at $\bar{\alpha}=\frac{\hat{v}_{y}^{2}}{4 \bar{K}}$.

The patterns developing during the very early stages of the evolution, i.e. when the nonlinear terms are still negligible, correspond to ripples built on the most unstable wave vector. According to the values of $\left|\hat{v}_{x}\right|$ and $\left|\hat{v}_{y}\right|$ the selected wavelength is thus:

$$
\lambda_{i}=\frac{2 \pi}{q_{i}}=2 \pi\left(\frac{2 \bar{K}}{\left|\hat{v}_{i}\right|}\right)^{\frac{1}{2}}
$$

The resulting wavelength expression is very parameter sensitive, meaning that the scale of the selected wavelength will depend significantly on the variables that determine the diffusive coefficient $\bar{K}$ and the erosive coefficient $\hat{v}_{x}$. Figure 2 illustrates the wavelength scale at various values of temperature, which is inversely proportional to $\bar{K}$, as well as increasing values of ion flux, $J$, which is directly proportional to the erosive coefficient. The ion and material parameters were designed to represent the bombardment of $\mathrm{Ar}^{+}$on tungsten. It can be seen that the scale ranges from the nanometer to the millimeter range, depending on the magnitude of ion flux and temperature. Other parameters, including ion energy $\epsilon$, penetration depth $a$, and the dimensions of the collision cascade will likewise affect the erosive contribution, while material properties such as the surface diffusivity and atomic density will affect the diffusive contribution. For the general case where $\bar{K}$ is

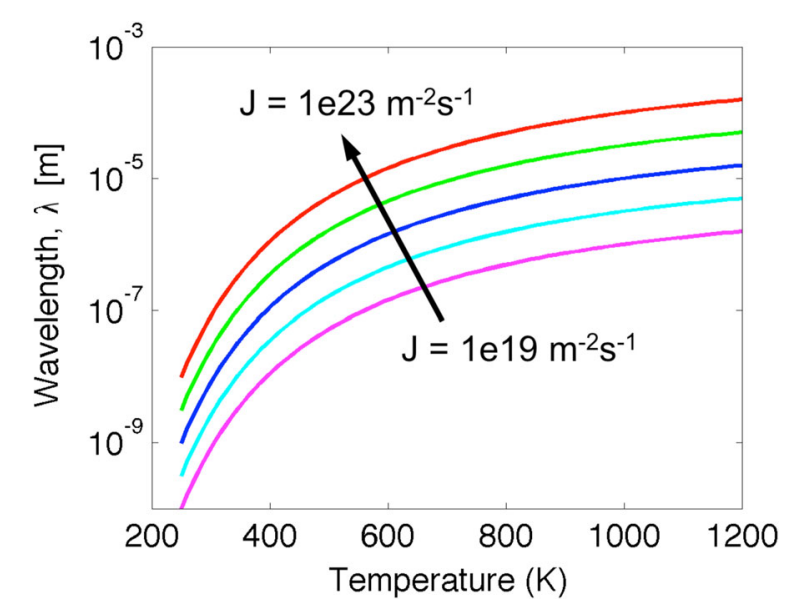

Fig. 2 Dependence of the ripple wavelength on temperature for various incident ion flux $\mathrm{J}$ values, from $10^{19}$ to $10^{23} \mathrm{~m}^{-2} \mathrm{~s}^{-1} \cdot D_{0}=2.4025 \times 10^{-7} \mathrm{~m}^{-2}, \gamma=2.9[\mathrm{~J}] /[\mathrm{m}]^{2}, \Omega=1.5825 \times 10^{-29} \mathrm{~m}^{3}, \rho_{\mathrm{s}}=7.0811 \times 10^{18}$ $\mathrm{m}^{-2}, \varepsilon=300 \mathrm{eV}$, normal ion incidence. The scale of the resulting wavelength is shown to be greatly affected by the ion flux $J$ and the temperature 
also anisotropic, the determination of the linearly selected patterns is more complicated, as shown by Bradley and Harper (1988).

The range of ripple sizes observed in plasma-facing materials has been well documented. As previously reviewed, nano-ripples were observed on glass substrates early on by Navez et al. (1962). In addition, ripple formations have been seen to develop on the insulator rings of Hall thrusters at the mm-scale (De Grys et al. 2010). The latter case likely involved the influence of stress effects due to plasma bombardment and thermal conditions. These experimental observations demonstrate the variety of size scales that may result from plasma exposure as surface features develop.

\section{Weakly nonlinear stability analysis}

As time proceeds, nonlinear terms grow and have to be taken into account. According to the distance from the instability threshold, these terms may saturate the linear growth of unstable modes and stabilize specific spatial patterns, or may induce spatio-temporal behavior that is irregular in space and time. To discuss the qualitative aspects of these regimes, let us consider the damping rate $\alpha$ as a varying parameter with other coefficients corresponding to anisotropies, diffusion, beam orientation, etc. as fixed.

For $\theta$ such that $\left|\hat{v}_{x}\right| \geq\left|\hat{v}_{y}\right|$ and $\bar{K}>1$. Equation 7 may be written to include the nonlinear term such that in Fourier transform, it is expressed as a convolution integral. Equation 7 in then written in Fourier space as:

$$
\begin{aligned}
\partial_{\tau} \bar{h}(\vec{q})= & -\left(\bar{\alpha}-\left|\hat{v}_{x}\right| q_{x}^{2}-\left|\hat{v}_{y}\right| q_{y}^{2}+q^{4} \bar{K}\right) \bar{h}(\vec{q}) \\
& -\int d \vec{k}\left[\hat{v}_{x}(q-k)_{x} k_{x}+\hat{v}_{y}(q-k)_{y} k_{y}\right] \bar{h}(\vec{q}-\vec{k}) \bar{h}(\vec{k}) \\
= & {\left[\epsilon-\bar{K}\left(q^{2}-q_{c}^{2}\right)^{2}-\left(\left|\hat{v}_{x}\right|-\left|\hat{v}_{y}\right|\right) q_{y}^{2}\right] \bar{h}(\vec{q}) } \\
& -\int d \vec{k}\left[\hat{v}_{x}(q-k)_{x} k_{x}+\hat{v}_{y}(q-k)_{y} k_{y}\right] \bar{h}(\vec{q}-\vec{k}) \bar{h}(\vec{k})
\end{aligned}
$$

where $q_{c}^{2}=\frac{\left|\hat{v}_{x}\right|}{2 \bar{K}}, \epsilon=\frac{\hat{v}_{x}^{2}}{4 \bar{K}}-\bar{\alpha}$. Hence, on decreasing $\bar{\alpha}$ below $\frac{\hat{v}_{x}^{2}}{4 \bar{K}}$, spatial modes with $\vec{q}= \pm q_{c} \overrightarrow{1}_{x}$ become first unstable, followed by modes different in wavenumber or in increasing orientation difference with $\overrightarrow{1}_{x}$ up to $\bar{\alpha}=\frac{\hat{v}_{y}^{2}}{4 \bar{K}}$, where modes with $\vec{q}= \pm \frac{\left|\hat{v}_{x}\right|}{2 \bar{K}} \overrightarrow{1}_{y}$ become unstable. For $\bar{\alpha}<\frac{\hat{v}_{y}^{2}}{4 \bar{K}}$, modes with all orientations become thus unstable, but the maximum growth rate still corresponds to $q_{c} \overrightarrow{1}_{x}$. Finally, for $\bar{\alpha}=0$, the set of unstable wave vectors extends to $\vec{q}=0$, characteristic of the undamped KS equation.

Let us consider first small values of $\epsilon\left(\bar{\alpha}\right.$ slightly below $\left.\frac{\hat{v}_{x}^{2}}{4 \bar{K}}\right)$. In this case, the range of unstable wave vectors is defined by $q_{c}^{2}-\sqrt{\frac{\epsilon}{K}}<q^{2}<q_{c}^{2}+\sqrt{\frac{\epsilon}{K}}$. For small $\epsilon$, corresponding to the weakly nonlinear regime, stable modes may be adiabatically eliminated and amplitude equations may be derived (Ghoniem and Walgraef 2008).

- For example, for critical ripples of uniform amplitude $\left(\bar{h}=A_{1} e^{i q_{c} x}+A_{2} e^{i 2 q_{c} x}+\ldots+c . c\right)$ one obtains:

$$
\begin{aligned}
& \partial_{t} A_{1}=\epsilon A_{1}+4 q_{c}^{2} \nu_{x} A_{2} A_{1}^{*}+\ldots \\
& \partial_{t} A_{2}=\left(\epsilon-9 K q_{c}^{4}\right) A_{2}-q_{c}^{2} \nu_{x} A_{1} A_{1}+\ldots
\end{aligned}
$$

and the adiabatic elimination of harmonics leads to

$$
\partial_{t} A_{1}=\epsilon A_{1}-u\left|A_{1}\right|^{2} A_{1}+\ldots
$$


where $u=\frac{4 q_{c}^{4} v 2}{K q_{c}^{4}-\epsilon} \simeq \frac{4 v_{x}^{2}}{K}$. Similarly, amplitude equations for spatially varying amplitude may be obtained within the usual methods of pattern formation theory (Ghoniem and Walgraef 2008; Walgraef 1997), resulting in the formation of stable ripples with critical wave vector parallel the $x$ axis and amplitudes $\left|A_{1}\right|=\sqrt{\frac{\epsilon}{u}}$, $\left|A_{2}\right|=\epsilon, \ldots$

- The quadratic nonlinearity also couples triplets of modes satisfying the triangular relation $\vec{q}_{1}+\vec{q}_{2}+\vec{q}_{3}=0$ which are able to destabilize one-dimensional patterns and sustain stable hexagonal ones. For example, the wave vector of critical ripples, considered as $\vec{q}_{1}=q_{c} \overrightarrow{1}_{x}$, is coupled to $\vec{q}_{2}=-\frac{q_{c}}{2} \overrightarrow{1}_{x}+\frac{\sqrt{3}}{2} q_{c} \overrightarrow{1}_{y}$ and $\vec{q}_{3}=-\frac{q_{c}}{2} \overrightarrow{1}_{x}-\frac{\sqrt{3}}{2} q_{c} \overrightarrow{1}_{y}$. On writing the profile built on these wave vectors as $\bar{h}=\sum_{n}\left(A_{n} e^{i n \vec{q}_{1} \vec{r}}+B_{n} e^{i n \vec{q}_{2} \vec{r}}+C_{n} e^{i n \vec{q}_{3} \vec{r}}+c . c\right)$, the corresponding amplitude equations, for the dominant contributions, have the structure:

$$
\begin{aligned}
& \dot{A}_{1}=\epsilon A_{1}+v_{A} B^{*} C^{*}-u_{A}\left|A_{1}\right|^{2} A_{1} \\
& \dot{B}_{1}=(\epsilon-\Delta) B_{1}+v_{B} A_{1}^{*} C_{1}^{*}-u_{B}\left|B_{1}\right|^{2} B_{1} \\
& \dot{C}_{1}=(\epsilon-\Delta) C_{1}+v_{C} B_{1}^{*} A_{1}^{*}-u_{C}\left|C_{1}\right|^{2} C_{1}
\end{aligned}
$$

where $\Delta=\frac{3}{4} q_{c}^{2}\left(\left|\hat{v}_{x}\right|-\left|\hat{v}_{y}\right|\right), v_{A}=\frac{q_{c}^{2}}{2}\left(3 \hat{v}_{y}-\hat{v}_{x}\right), v_{B}=v_{C}=q_{c}^{2} \hat{v}_{x}, u_{A}=\frac{4 \hat{v}_{x}^{2}}{9 \bar{K}}$, $u_{B}=u_{C}=\frac{4}{9 \bar{K}+4 \Delta}\left(\frac{\left(\hat{v}_{x}-3 \hat{v}_{y}\right)^{2}}{4}\right)$. Critical ripples correspond to the steady state $\left|A_{1}\right|_{s}=\sqrt{\frac{\epsilon}{u_{A}}}, B_{1}=C_{1}=0$. The linear evolution of perturbations of this state is given by:

$$
\begin{aligned}
& \dot{B}_{1}=(\epsilon-\Delta) B_{1}+v_{B} A_{1 s}^{*} C_{1}^{*} \\
& \dot{C}_{1}=(\epsilon-\Delta) C_{1}+v_{C} B_{1}^{*} A_{1 s}^{*}
\end{aligned}
$$

and the corresponding linear growth rate is positive for

$$
\epsilon>\frac{v_{B}^{2}}{4 u_{A}}\left[-1+\sqrt{1+\frac{4 \Delta u_{A}}{v_{B}^{2}}}\right]^{2}=\frac{9 \bar{K} q_{c}^{4}}{16}\left[-1+\sqrt{1+\frac{16 \Delta}{9 \bar{K} q_{c}^{4}}}\right]^{2}=\epsilon_{c}
$$

Hence, in large systems, for which the present discussion is valid, when $\epsilon<\epsilon_{c}$, ripples should be selected, while for $\epsilon>\epsilon_{c}$, steady state solutions of (16), corresponding to anisotropic hexagonal patterns (also viewed as "dots-on-ripples" patterns), should develop.

Note that in the two-variable model proposed by Motta et al. $(2012,2014)$, the resulting set of unstable wave vectors consists of a finite domain around the critical wave vector, as in the DKS equation, plus a marginal mode with $\vec{q}=0$. This is a classical problem of Turing-like instability coupled with a Goldstone mode (Cox and Matthews 2003; Dewel et al. 1995). The nonlinear couplings between the Goldstone mode and the finite wavelength unstable modes induce differences in the selection and stability ranges of patterns. In reference (Motta et al. 2012), a detailed amplitude equation analysis, including the effect of anisotropy, leads to a similar conclusion also supported by numerical analysis. The comparison between the outcome of the DKS and the two-variable model would of course be highly desirable in the analysis of specific experimental situations. 


\section{Beyond the weakly nonlinear regime}

By decreasing the damping rate $\alpha$ (increasing $\epsilon$ ), the range of unstable wave vectors increases, which allows more coupling between small and large scales. The weakly nonlinear approximation breaks down and the amplitude equation description is not valid anymore. Spatio-temporal patterns usually develop and numerical analysis is required to study them, as we will present in the Numerical simulations of pattern evolution Section. However, for vanishing damping, our evolution equation becomes a genuine anisotropic KS equation. While the one-dimensional KS equation has been studied in detail in a huge number of papers (a few basic references are (Cvitanovíc et al. 2010; Hyman and Nicolaenko 1986; Hyman et al. 1986; Kevrekidis et al. 1990)), the 2D version, which in the undamped case has features of the KPZ equation, is much less investigated. In the damped case, the formation of steady hexagonal patterns, breathing hexagons or more complex spatio-temporal behavior has been reported (Gomez and Paris 2011; Paniconi and Elder 1997). In the $2 \mathrm{D}$ anisotropic case, coarsening ripples may appear in the undamped case (Rost and Krug 1995), while in the damped case, a numerical study shows competition between one- and two-dimensional patterns (Vitral 2015).

Let us just recall here very basic aspects of the problem which are relevant to the present investigation.

- One dimension. For values of $\theta$ such that $\left|\hat{v}_{x}\right|>\left|\hat{v}_{y}\right|$, the system is anisotropic and its behavior may be considered as essentially one-dimensional, where the evolution equation is:

$$
\partial_{\tau} \bar{h}=-\left|\hat{v}_{x}\right| \bar{h}_{X X}-\left(\hat{D}_{x x}+\bar{K}\right) \bar{h}_{X X X X}+\hat{v}_{x}\left(\bar{h}_{X}\right)^{2}
$$

This equation is one of the simplest PDEs, which exhibits spatiotemporal chaotic behavior. When $x \in[0, L]$, Eq. (19) is equivalent to an infinite set of ODEs:

$$
\frac{d}{d t} \hat{h}_{k}=\left(\left|\hat{v}_{x}\right| k^{2}-\left(\hat{D}_{x x}+\bar{K}\right) k^{4}\right) \hat{h}_{k}-\hat{v}_{x} \Sigma_{k^{\prime}}\left(k-k^{\prime}\right) k^{\prime} \hat{h}_{k-k^{\prime}} \hat{h}_{k^{\prime}}
$$

with $\bar{h}(x, t)=i \Sigma_{k} \hat{h}_{k}(t) \exp (i k x), k=n q, q=\frac{2 \pi}{L}, n \in Z$. The zero solution is unstable versus modes with $|k|<\sqrt{\frac{\left|\hat{v}_{x}\right|}{\hat{D}_{x x}+\bar{K}}}=\frac{2 \pi}{L_{c}}$, or $L>L_{c}$ (the number of such modes increases with $L$ ). If $L$ is taken as the bifurcation parameter and grows beyond $L_{c}$, the solution passes through a complex hierarchy of bifurcations leading to cellular multi-modal stationary, oscillatory and chaotic states. A typical behavior includes an irregular succession of windows with quasi-periodic and chaotic behavior.

In this analysis, it is the boundary condition that determines the wavenumbers which enter in the unstable domain. In our problem, on varying beam orientation, temperature $(\bar{K})$, or damping rate, one may increase or decrease the number of unstable modes in the system and expect similar results as in the previously mentioned finite domain case. Following a high $T$ approximation, $\bar{K}$ dominates and $D_{x x}$ may be neglected (Bradley and Harper 1988; Makeev et al. 2002). In the absence of damping, the domain of unstable wave vectors extends to zero, which rules out an amplitude equation description, except, perhaps, for the first stages of the evolution, which is dominated by the fastest growing modes and the ones generated by nonlinear interactions. The evolution is described by Eq. (19) and the fastest growing mode corresponds to $\vec{q}_{0}=\sqrt{\frac{\left|\hat{v}_{x}\right|}{2 \bar{K}^{\prime}}} \overrightarrow{1}_{x}$ with a growth rate $q_{0}^{4} \bar{K}$. It is directly coupled, 
through the quadratic nonlinearity, with $2 \vec{q}_{0}, \frac{1}{2} \vec{q}_{0}$. The resulting evolution equations for these modes are:

$$
\begin{aligned}
\frac{d}{d t} \bar{h}\left(\vec{q}_{0}, t\right) & =\bar{K} q_{0}^{4} \bar{h}\left(\vec{q}_{0}, t\right)+4 q_{0}^{2} \hat{v}_{x} \bar{h}\left(2 \vec{q}_{0}, t\right) h\left(-\vec{q}_{0}, t\right)-\frac{q_{0}^{2}}{2} \hat{v}_{x} \bar{h}\left(\frac{\vec{q}_{0}}{2}, t\right)^{2} \\
\frac{d}{d t} \bar{h}\left(2 \vec{q}_{0}, t\right) & =-8 \bar{K} q_{0}^{4} \bar{h}\left(\overrightarrow{2} q_{0}, t\right)-q_{0}^{2} \hat{v}_{x} \bar{h}\left(\vec{q}_{0}, t\right)^{2}+\ldots \\
\frac{d}{d t} \bar{h}\left(\frac{ \pm \vec{q}_{0}}{2}, t\right) & =\frac{7}{16} \bar{K} q_{0}^{4} \bar{h}\left(\frac{ \pm \vec{q}_{0}}{2}, t\right)+q_{0}^{2} \hat{v}_{x} \bar{h}\left(\vec{q}_{0}, t\right) \bar{h}\left(\mp \frac{\vec{q}_{0}}{2}, t\right)
\end{aligned}
$$

$\bar{h}\left(2 \vec{q}_{0}, t\right)$ may be adiabatically eliminated and one obtains:

$$
\begin{gathered}
\frac{d}{d t} \bar{h}\left(\vec{q}_{0}, t\right)=\bar{K} q_{0}^{4} \bar{h}\left(\vec{q}_{0}, t\right)-\frac{\hat{v}_{x}^{2}}{2 \bar{K}} \bar{h}\left(\vec{q}_{0}, t\right)^{2} h\left(-\vec{q}_{0}, t\right)-\frac{q_{0}^{2} \hat{v}_{x}}{2} \bar{h}\left(\frac{\vec{q}_{0}}{2}, t\right)^{2} \\
\frac{d}{d t} \bar{h}\left(\frac{ \pm \vec{q}_{0}}{2}, t\right)=\frac{7}{16} \bar{K} q_{0}^{4} \bar{h}\left(\frac{ \pm \vec{q}_{0}}{2}, t\right)-q_{0}^{2} \hat{v}_{x} \bar{h}\left(\vec{q}_{0}, t\right) \bar{h}\left(\mp \frac{\vec{q}_{0}}{2}, t\right)
\end{gathered}
$$

On the fastest time scale, $O\left(1 / \bar{K} q_{0}^{4}\right), \bar{h}\left(\vec{q}_{0}, t\right)$ saturates to $\frac{\sqrt{2} \bar{K} q_{0}^{2}}{\left|v_{x}\right|}$ with $\bar{h}\left( \pm \frac{\vec{q}_{0}}{2}\right)=0$. However this solution is always unstable versus $\bar{h}\left( \pm \frac{\vec{q}_{0}}{2}, t\right)$. Effectively, the linear evolution of small perturbations $\bar{h}\left( \pm \frac{\vec{q}_{0}}{2}, t\right)$ is given by:

$$
\begin{aligned}
\frac{d}{d t} \bar{h}\left(\frac{\vec{q}_{0}}{2}, t\right) & =\frac{7}{16} \bar{K} q_{0}^{4} \bar{h}\left(\frac{\vec{q}_{0}}{2}, t\right)+\sqrt{2} \bar{K} q_{0}^{4} \bar{h}\left(-\frac{\vec{q}_{0}}{2}, t\right) \\
\frac{d}{d t} \bar{h}\left(-\frac{\vec{q}_{0}}{2}, t\right) & =\frac{7}{16} \bar{K} q_{0}^{4} \bar{h}\left(-\frac{\vec{q}_{0}}{2}, t\right)+\sqrt{2} \bar{K} q_{0}^{4} \bar{h}\left(\frac{\vec{q}_{0}}{2}, t\right)
\end{aligned}
$$

Hence, the quadratic coupling between $\bar{h}\left(\vec{q}_{0}, t\right)$ and $\bar{h}\left( \pm \frac{\vec{q}_{0}}{2}, t\right)$ enhances the latter's growth rate to about $1.85 \bar{K} q_{0}^{4}$. A cellular state with wavelength $\lambda=\lambda_{0}=2 \pi / q_{0}$ grows thus first and, after some time, is replaced by another cellular state with $\lambda=2 \lambda_{0}$. This situation has been studied in more detail by Misbah et al. in (Misbah and Valance 1994). Similar arguments may be performed for successive subharmonics and provide a qualitative picture for the first stages of the surface evolution.

- Two dimensions. In this case, the fastest growing mode remains the mode with $\vec{q}=\vec{q}_{0}=q_{0} \overrightarrow{1}_{x}$. Besides the quadratic coupling with $2 \vec{q}_{0}$ and $\frac{1}{2} \vec{q}_{0}, \vec{h}\left(\vec{q}_{0}, t\right)$ may also be coupled with $\bar{h}\left(\vec{q}_{1}, t\right)$ and $\bar{h}\left(\vec{q}_{2}, t\right)$ where $\vec{q}_{0}=q_{0} \overrightarrow{1}_{x}, \vec{q}_{1}=-\frac{1}{2} q_{0} \overrightarrow{1}_{x}+\frac{\sqrt{3}}{2} q_{0} \overrightarrow{1}_{y}$, $\vec{q}_{2}=-\frac{1}{2} q_{0} \overrightarrow{1}_{x}-\frac{\sqrt{3}}{2} q_{0} \overrightarrow{1}_{y}$. The coupled evolution of these modes is then:

$$
\begin{aligned}
& \frac{d}{d t} \bar{h}\left(\vec{q}_{1}, t\right)=\left(\bar{K} q_{0}^{4}-\Delta\right) \bar{h}\left(\vec{q}_{1}, t\right)+q_{0}^{2} \hat{v}_{x} \bar{h}\left(-\vec{q}_{0}, t\right) \bar{h}\left(-\vec{q}_{2}, t\right)+\ldots \\
& \frac{d}{d t} \bar{h}\left(\vec{q}_{2}, t\right)=\left(\bar{K} q_{0}^{4}-\Delta\right) \bar{h}\left(\vec{q}_{2}, t\right)+q_{0}^{2} \hat{v}_{x} \bar{h}\left(-\vec{q}_{0}, t\right) \bar{h}\left(-\vec{q}_{1}, t\right)+\ldots
\end{aligned}
$$

where $\Delta=\frac{3}{4} q_{0}^{2}\left(\left|\hat{v}_{x}\right|-\left|\hat{v}_{y}\right|\right)$. On the saturation scale of $\bar{h}\left(\vec{q}_{0}, t\right)$, the maximum growth rate of $\bar{h}\left(\vec{q}_{1,2}, t\right)$ is $\left((\sqrt{2}-0.5)\left|\hat{v}_{x}\right|+1.5|| \hat{v}_{y} \mid\right) \frac{q_{0}^{2}}{2}$.

This growth rate is greater than the effective growth rate of subharmonics $\left(1.85 \bar{K} q_{0}^{4}=1.85\left|\hat{v}_{x}\right| \frac{q_{0}^{2}}{2}\right)$ for $\left|\hat{v}_{x}\right|<1.6\left|\hat{v}_{y}\right|$. Hence, there is a range of incidence angles around $\theta=0$ where two-dimensional effects should dominate over subharmonic ones during the first stages of the evolution. Further analysis requires of course numerical simulations, as presented in the next section. 
Let us recall that we considered, in this section, systems with large spatial extension, where boundary effects are irrelevant, and random initial conditions. Boundary effects in small systems and preexisting initial patterns may force spatial modes into the system able to compete with the dynamically selected ones, and affect pattern selection and evolution. We consider these effects in a separate publication D Walgraef, CSR Matthes, NM Ghoniem: The influence of surface architecture on ion-induced nano-patterning, in preparation.

\section{Numerical simulations of pattern evolution}

\section{Method development}

To simulate surface evolution according to defined parameters, the evolution equation is solved numerically in Fourier space (D Walgraef: Nano-patterning of surfaces by ion sputtering. Working comments and thoughts, unpublished). If $u(x)$ is a function sampled at $n$ discrete points $x_{i} \in h, 2 h, \ldots, i h, \ldots, 2 \pi-h, 2 \pi$ and $h=2 \pi / n$ in real space, then the Fast Fourier Transform (FFT) is expressed as:

$$
\operatorname{FFT}\left(u_{j}\right) \equiv \hat{u}_{k} \quad \text { where } k \in\left(\frac{-n}{2}+1\right), \ldots, \frac{n}{2}
$$

The Fourier transform of derivatives can easily be found subsequently from $\hat{u}_{k}$ : $\operatorname{FFT}\left(\frac{\partial^{v} u_{j}}{\partial x^{v}}\right) \equiv(i k)^{v} \hat{u}_{k}$. To model the directional dependence of the expression, it is necessary to introduce the indices $k_{x}$ and $k_{y}$, as the derivatives are applied across both dimensions.

The nonlinear term of the evolution equation can be written in Fourier space using the convolution property. It should be noted that the convolution of two vectors in Fourier space (note the use of the convolution operator $[*]$ ) is equal to the Fourier transform of their element-wise product in real space. The derivatives are expressed as the product of $\hat{h}$ with powers of the index $k$. Damping can be added by taking into account translational invariance, achieved by subtracting the average surface height at each time step $h_{A}$ from the height profile $\hat{h}$. Thus,

$$
\begin{aligned}
\frac{\partial \hat{h}}{\partial t}= & -\alpha\left(\hat{h}-h_{A}\right)-\frac{F a}{2}\left(\left(i k_{x}\right)^{2} \hat{h}+\left(i k_{y}\right)^{2} \hat{h}\right)-\frac{F a_{\beta}^{2}}{2}\left(\hat{v}_{x}\left[\left(i k_{x}\right) \hat{h}\right] *\left[\left(i k_{x}\right) \hat{h}\right]\right. \\
& \left.+\hat{v}_{y}\left[\left(i k_{y}\right) \hat{h}\right] *\left[\left(i k_{y}\right) \hat{h}\right]\right)-K\left(\left(i k_{x}\right)^{4} \hat{h}+\left(i k_{y}\right)^{4} \hat{h}+2\left(i k_{x}\right)^{2}\left(i k_{y}\right)^{2} \hat{h}\right) .
\end{aligned}
$$

The nonlinear term requires the solution of the ODE to be performed using a hybrid implicit/explicit method, in which all terms are written implicitly except the nonlinear term, which is written explicitly. The result may be calculated in Fourier space for each time step $(n)$, then converted back to real space. $\hat{h}^{n+1}$ is the height in Fourier space at time step $n+1$, and $\Delta t$ is the time step increment.

$$
\hat{h}^{n+1}=\frac{\left[\hat{h}^{n}+\Delta t \alpha h_{A}-\Delta t \frac{F a_{\beta}^{2}}{2}\left(\hat{v}_{x}\left[\left(i k_{x}\right) \hat{h}^{n}\right] *\left[\left(i k_{x}\right) \hat{h}^{n}\right]+\hat{v}_{y}\left[\left(i k_{y}\right) \hat{h}^{n}\right] *\left[\left(i k_{y}\right) \hat{h}^{n}\right]\right)\right]}{\left[1+\Delta t \alpha+\Delta t \frac{F a}{2}\left[\left(i k_{x}\right)^{2}+\left(i k_{y}\right)^{2}\right]+\Delta t K\left[\left(i k_{x}\right)^{4}+\left(i k_{y}\right)^{4}+2\left(i k_{x}\right)^{2}\left(i k_{y}\right)^{2}\right]\right]}
$$

This result may be calculated for all time steps, and converted back to real space. The operation is performed over many time steps to simulate the evolution of the surface 
profile over a specified span. In the case of linear evolution, the nonlinear term in the numerator, expressed by the convolution, may be removed.

\section{Convergence and accuracy tests}

In this section, we address the accuracy and convergence of the numerical solutions. For this purpose, the "average" wavelength is used to help quantify the evolution of the surface according to the spectral density, $p$, of all emergent wavelengths in the spectrum. Figures 3 and 4 show the results of the evolving ripple morphology using the linear evolution equation for $\theta=30^{\circ}$, and an initial surface of $h=A \cos (\omega x) \sin (\omega y)$ where $\omega=3.2$. The evolution was carried out according to the parameters defined in Table 1.

The wavelength spectrum can by averaged at each time step in the following way: $\lambda_{\text {ave }}=$ $\frac{\Sigma\left(\lambda_{i} \cdot p_{i}\right)}{\Sigma \lambda_{i}}$, and is shown over a range of time steps on the left in Figs. 3 and 4 in the $x-$ and $y$-direction, respectively. It can be observed that the average wavelength begins at a value corresponding to the initial surface, and grows to a steady-state value as the surface morphology changes, representing the final "evolved" wavelength spectrum of the surface. The behavior of the average wavelength demonstrates the emergence of surface ripples of a particular wavelength, which cause the average spectral wavelength to grow toward a converged result as the most unstable wavelength takes over the morphology.

The "dominant" wavelength refers to the single wavelength with the highest spectral density at any given time. This value is shown on the right side in Figs. 3 and 4, and is observed to begin at a particular value determined by the initial configuration. In this case the dominant $\lambda$ is shown to switch after $9 \mathrm{~h}$ of simulated exposure to a value close to the analytical result, indicated by the horizontal black line. The steady-state value corresponding to the wavelength expected by linear stability analysis further indicates an accurate converged value.

The accuracy of the simulation is dependent on the number of discrete points that the surface is sampled at, defined by the grid size $n$, which corresponds to the FFT indices $k_{x}$ and $k_{y}$. Figure 5 shows the global error, or the difference between the final converged wavelength, and the analytical result, for different grid sizes. The global error is computed over grid sizes that correspond to increasing powers of 2, to optimize speed. It can be seen that for a sufficiently large grid size, the error reaches a steady value of $1.28 \times 10^{-6}$ $\mathrm{m}$ in the $\mathrm{x}$-direction, and $1.48 \times 10^{-6} \mathrm{~m}$ in the $\mathrm{y}$-direction. Relative to the scale of the
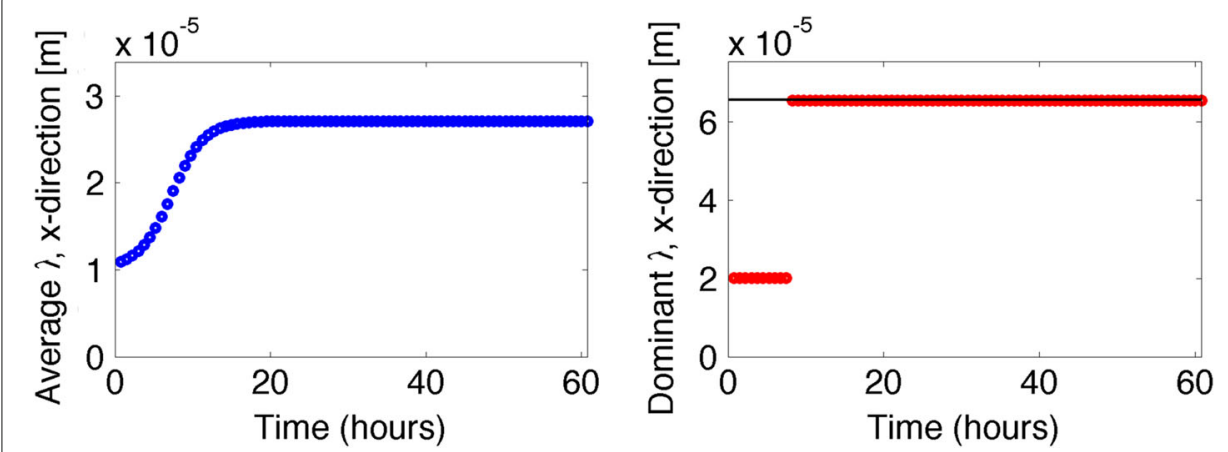

Fig. 3 Convergence of the averaged wavelength in the $x$-direction (left) to show changes in the distribution function over time. The dominant wavelength (right) shows the ripple wavelength that is most present in the morphology. Black horizontal line indicates analytical result 

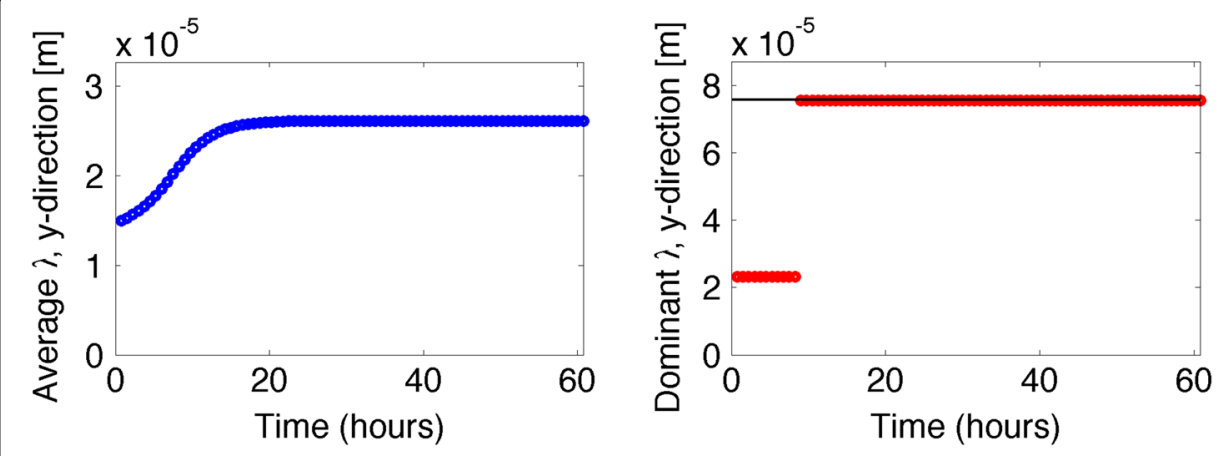

Fig. 4 Convergence of the averaged wavelength in the $y$-direction (left) to show changes in the distribution function over time. The dominant wavelength (right) shows the ripple wavelength that is most present in the morphology. Black horizontal line indicates analytical result

surface features, where the dominant wavelength is on the order of $10^{-4} \mathrm{~m}$, this error is sufficiently low.

\section{Numerical simulation results}

Numerical simulations using the FFT method were performed to provide insight into the long-term evolution of the surface height, and extend the scope of analytical predictions described above. The simulations were performed without the presence of damping (i.e. $\bar{\alpha}=0$ ), such that the bifurcation parameter $\epsilon$, and thus the range of unstable wave vectors is maximized. Under these conditions, surface symmetries according to the linear evolution equation may not match directly with theory, and pattern formation according to weakly nonlinear analysis becomes less predictable. Periodic boundary conditions have been applied. Spectral analysis was performed on the surface profiles to allow for a determination of dominant frequencies associated with the ripple wavelengths present in the developed symmetries. The parameter values used in all of the present numerical calculations are given in Table 1.

\section{Effects of nonlinearities in the transient regime}

The quadratic non-linearity introduced in the surface evolution equations is physically a result of large local geometric variations, in violation of the small slope approximation of the original $\mathrm{BH}$ approach. Therefore, one expects that as time proceeds, the influence of higher order non-linearities will become more significant. Our previous analytical results indicated that couplings between the dominant surface mode and other modes that are

Table 1 Parameters used to define the surface evolution

\begin{tabular}{llll}
\hline Parameter & Description & Quantity & Units \\
\hline$J$ & Ion flux & $5 \times 10^{21}$ & {$[\mathrm{~m}]^{-2}[\mathrm{~s}]^{-1}$} \\
$\varepsilon$ & Ion energy & 300 & {$[\mathrm{eV}]$} \\
$a$ & Ion penetration depth & $2 \times 10^{-9}$ & {$[\mathrm{~m}]$} \\
$\beta$ & Collision cascade dimension & $1 \times 10^{-9}$ & {$[\mathrm{~m}]$} \\
$D_{0}$ & Surface diffusivity & $2.4025 \times 10^{-7}$ & {$[\mathrm{~m}]^{-2}$} \\
$\gamma$ & Surface free energy density & 2.9 & {$[\mathrm{~J}] /[\mathrm{m}]^{-2}$} \\
$\Omega$ & Atomic volume & $1.5825 \times 10^{-29}$ & {$[\mathrm{~m}]^{3}$} \\
$\rho_{S}$ & Atomic surface density & $7.0811 \times 10^{18}$ & {$[\mathrm{~m}]^{-2}$} \\
$T$ & Temperature & 500 & {$[\mathrm{~K}]$} \\
\hline
\end{tabular}



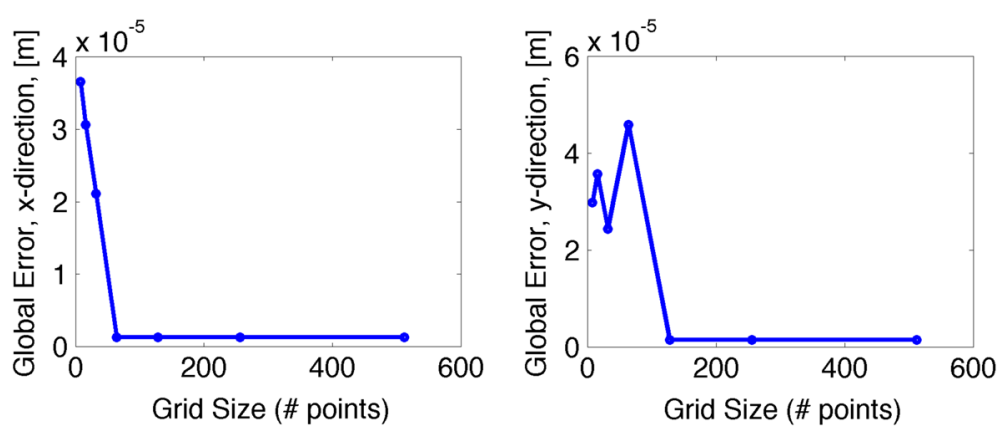

Fig. 5 Global error for various grid sizes in the x-direction (left) and y-direction (right). The error is shown to converge to a minimum with a grid size of 128

twice and half of the dominant wavelength will appear. Moreover, if the initial surface is intentionally structured, as previous experimental efforts have done using Chemical Vapor Deposition ( $\mathrm{Li}$ et al. 2017; Matthes et al. 2017), it would be interesting to see the role of non-linearities in pattern selection. A full exploration of the relationship between the intial surface architecture and dynamically selected pattern is presented in reference D Walgraef, CSR Matthes, NM Ghoniem: The influence of surface architecture on ioninduced nano-patterning, in preparation. Here, we consider a case of an initial structured surface and numerically follow its linear and non-linear evolution.

The observed nonlinear patterning is explained visually in Fig. 6a. The top plot shows the initial pattern, composed of a series of 4 gaussian "bump" formations. This initial architecture was selected because it resulted in an increased speed of pattern selection compared to a randomized initial profile. Using this initial surface geometry, the nonlinear wavelength emerges in the profile after about $30 \mathrm{~h}$, rather than closer to $75 \mathrm{~h}$ for a randomized configuration. At an ion flux of $5 \times 10^{21} \mathrm{~m}^{-2} \mathrm{~s}^{-1}$, this corresponds to ion

a

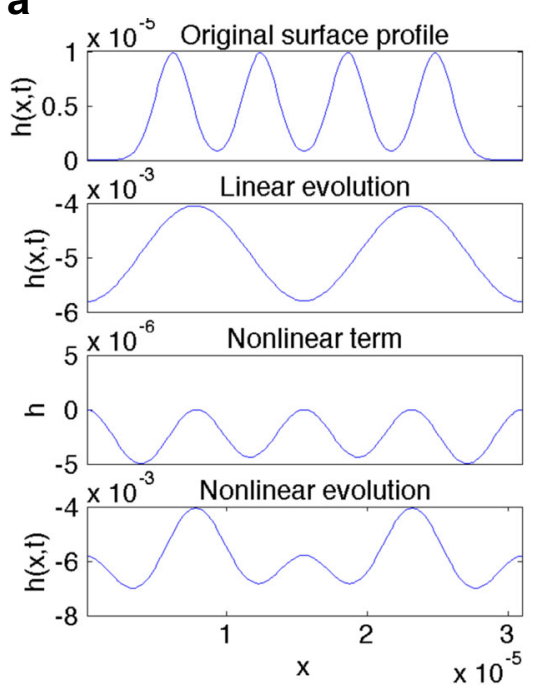

b
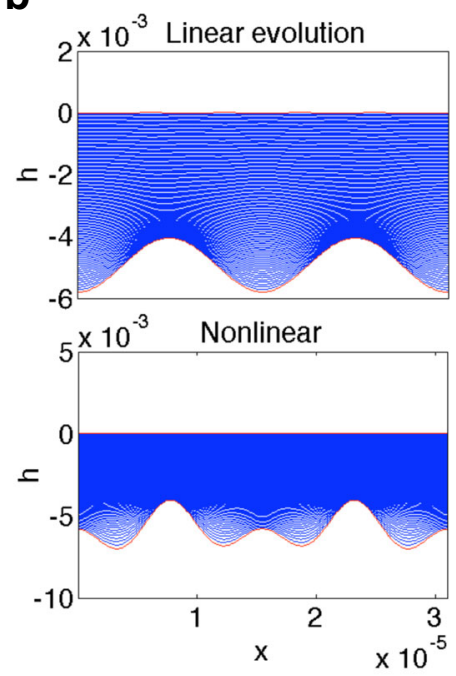

Fig. 6 a Comparison of the initial surface with the final surface profiles under both linear and nonlinear evolution, with nonlinear term plotted to show the wavelength magnitude. The original surface is a series of 4 gaussian bumps. b Evolution of a gaussian bump surface profile using the linear form of the evolution equation (top) and nonlinear term included (bottom), showing the influence of the nonlinear term. All units shown are in $[\mathrm{m}]$ 
fluences of $5.4 \times 10^{26} \mathrm{~m}^{-2}$ and $8.1 \times 10^{26} \mathrm{~m}^{-2}$, respectively. The next plot down shows the periodic pattern of the evolved surface profile, with a wavelength that corresponds to linear stability analysis of the evolution equation. The plot below the linearly evolved profile shows the isolated nonlinear term, which clearly has twice the frequency of the linearly evolved profile. When factored into the evolution equation, the effect nonlinear term is magnified as it grows, and plays a large part in the evolved surface profile. With this nonlinear contribution, it can be seen how the patterning of the nonlinear evolution comes about. Figure $6 \mathrm{~b}$ shows the 1-dimensional evolution of a particular surface for both the linear and nonlinear case after $34 \mathrm{~h}$ of exposure. It can be seen that the nonlinear plot possesses similar periodicity to the linear plot, but with additional undulations in the final profile. It should be noted that while the linear profile is steady-state, the nonlinear profile is at a transient point in its morphological evolution (i.e. the final converged symmetry is not yet reached for the chosen time of the simulation). This point of evolution was selected to observe the combined effect of the linear pattern selection with the added effect of the nonlinear term prior to it dominating the morphology. At later times, the nonlinear term takes over, and the morphology is dictated by the corresponding wavelength alone.

The spectral analysis shown in Fig. 7 corresponds to the evolution shown in Fig. 6. It can be seen that prior to evolution, the original surface possesses a distinct pattern, which disappears after $30 \mathrm{~h}$ of evolution in both the linear and nonlinear case. Under linear evolution, it can be seen that a peak exists close to the analytically expected result for linear stability, denoted by the black vertical line. A notable observation is that the evolved geometry for the nonlinear case has a dominant wavelength present that is approximately half the size of the analytical result. There also exists a peak on the nonlinear plot near the analytical result, indicating the presence of the linear morphology in the final patterning, as can be observed in the plot from Fig. 7. An intermediate wavelength spectrum is also displayed, taken after $10 \mathrm{~h}$ of exposure, and from this it can be observed that the dominant

Linear Evolution
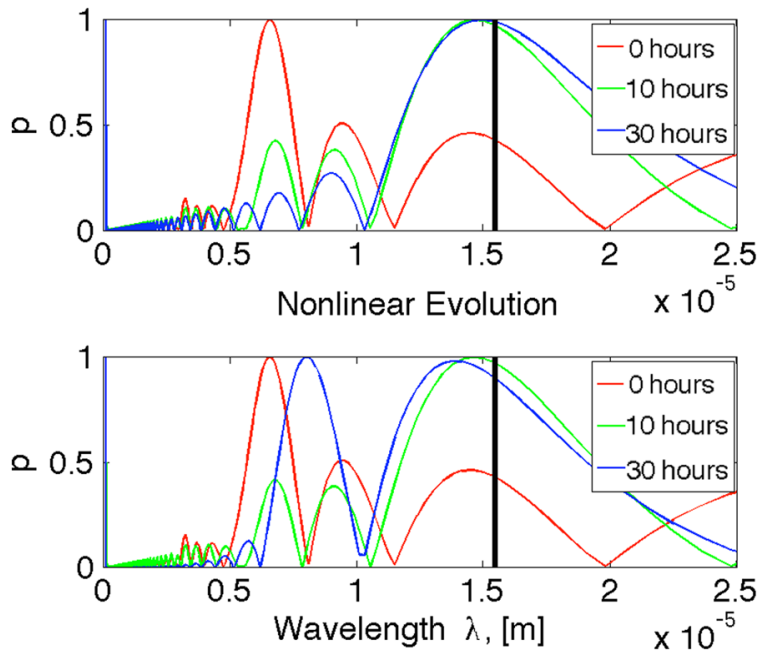

Fig. 7 Wavelength spectrum for linear (top) and nonlinear (bottom) evolution cases. The distribution function of the surface is shown prior to evolution, after $10 \mathrm{~h}$, and after $30 \mathrm{~h}$ to demonstrate the changes throughout evolution. The effect of nonlinearities shows the emergence of a wavelength at later times, which is half the analytical result according to linear theory. After 10 hours, both evolution regimes show similar results 
wavelength for both linear and nonlinear evolution were identical, close to the analytical result. This indicates that at earlier time steps, the nonlinear term has little effect, as it has not grown sufficiently to influence the morphology.

\section{System behavior in 2 dimensions}

Figure 8 shows the surface color plots corresponding to the surface height, in $\mathrm{m}$, of a surface that has a randomized initial height profile (shown as pixels in Fig. 8a), and the same surface shown after $60 \mathrm{~h}$ of simulated exposure at normal ion incidence $(\theta=0)$, for both linear (Fig. 8b) and nonlinear (Fig. 8c) evolution. Here we assume no effects due to damping (i.e. $\alpha=0$ ). The red color areas indicate surface peaks, while the blue color areas correspond to troughs.

It can be observed that when using the linear equation, the evolved surface (Fig. 8b) demonstrates the emergence of "dots-on-ripples" type patterning, where organized ripples mixed with hexagonal features have been produced. The ripple formation corresponds to previous experimental work on graphite (Habenicht 2001) and glass (Navez et al. 1962) surfaces, as well as certain metals (Costantini et al. 2001; Valbusa et al. 2002). The ripples are oriented at an angle relative to the incident ions traveling in the $\overrightarrow{1}_{x}$ direction. Because the angle of incidence is set to the surface normal $(\theta=0)$, the curvature coefficients $\hat{v}_{x}$ and $\hat{v}_{y}$ are equal, such that orientational degeneracy is expected. The simulations displayed this degeneracy in the direction of the emergent ripples (i.e. the $\mathrm{x}$ - and $y$-components changed between positive and negative with successive simulations), but both components were shown to be equal in magnitude, as expected analytically. The wave vectors in the displayed case can be defined as $\vec{q}_{1}=q_{c} \overrightarrow{1}_{x}, \vec{q}_{2}=-\frac{q_{c}}{2} \overrightarrow{1}_{x}-\frac{q_{c}}{2} \overrightarrow{1}_{y}$, and $\vec{q}_{3}=-\frac{q_{c}}{2} \overrightarrow{1}_{x}+\frac{q_{c}}{2} \overrightarrow{1}_{y}$, satisfying the triangular relation $\vec{q}_{1}+\vec{q}_{2}+\vec{q}_{3}=0$. The linear result demonstrates the presence of all three wave vectors, with more dominant critical ripples corresponding to $\vec{q}_{2}$, having components in both the $\mathrm{x}$ - and y-directions. In these simulations, we are operating in a regime where the damping rate $\alpha$ is set to zero, making $\epsilon$ a maximum value. Therefore, the evolution of the surface is determined according to a KPZ equation form. The result shows the emergence of a number of coupled unstable wave vectors, resulting in the "dots-on-ripples" pattern that has emerged. Successive simulations showed the orientation of the dominant ripples to vary between $\vec{q}_{2}$ and $\vec{q}_{3}$, as the randomized initial surface allowed for the competition between wave vectors to vary.

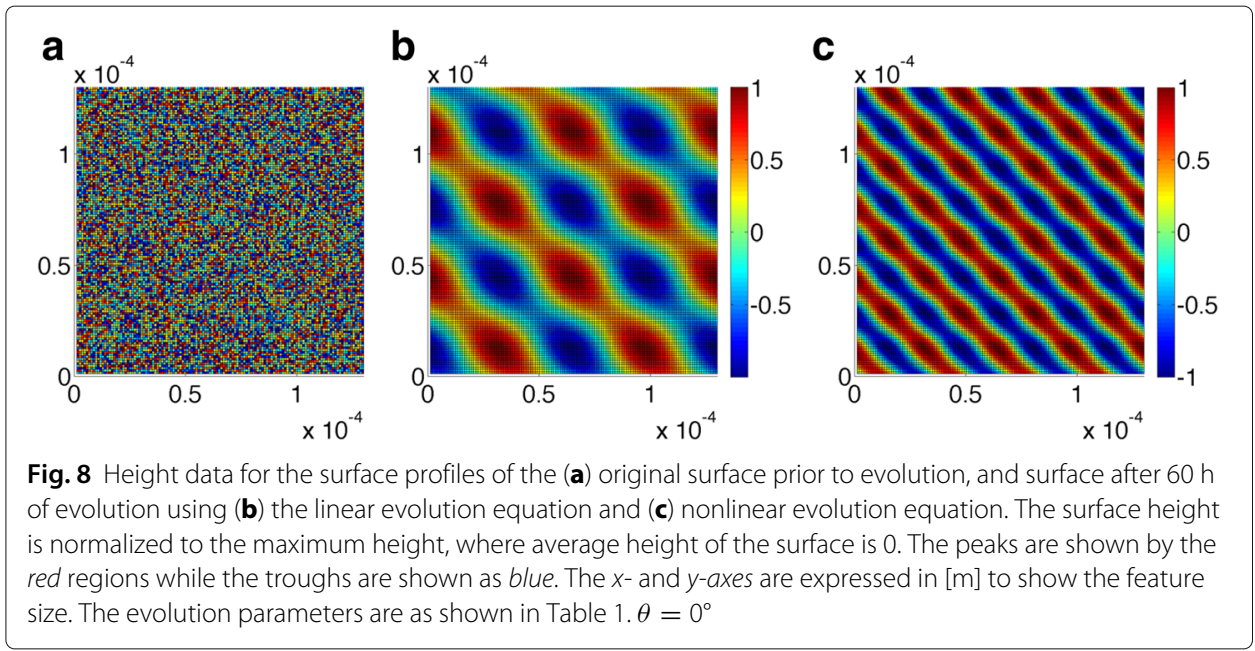


The evolution results according to the nonlinear equation (Fig. 8c) show the formation of striations similar to that of the linear evolution. However, the wavelength scale differs. It can be seen that the nonlinear evolution produced wavelengths that are noticeably smaller in size than the linear evolution, replicating the nonlinear behavior demonstrated in Fig. 6, where the wavelength is half the magnitude as the linear result. The orientation of the critical ripples, like the linear result, corresponded to the $\vec{q}_{2}$ mode, indicating the presence of other modes to be less consequential in determining the resulting symmetries for the shown simulation. In other simulations with a randomized initial profile, it was observed that the critical mode varied between $\vec{q}_{2}$ and $\vec{q}_{3}$, indicating a degenerate solution. The scale of the ripple wavelength appears to be half the magnitude of the wavelength in the linear case, indicating the emergence of $2 \vec{q}_{2}$ as the dominant wave vector, which was consistent for all simulations.

Patterning of the surface profile can also be investigated using Fourier spectral analysis, where the distribution function of the various surface modes (wavelengths) can give direct evidence of the regularity of emerging patterns. Figure 9 shows the wavelength spectrum of the initial surface profile (red line), shown together with the final surface profile spectrum (blue line) after $60 \mathrm{~h}$ of exposure, in both the linear (top) and nonlinear (bottom) case. The results are displayed in both the $\mathrm{x}$-direction (Fig. 9a) and y-direction (Fig. 9b). A vertical black line was added to the plots to show the value of the dominant wavelength obtained from the analytical solutions of the previous section. Both the $\mathrm{x}$ - and $\mathrm{y}$-directions show similar results in that the linear evolution produces a dominant wavelength, shown by the tallest peak, which is consistent with the analytical result. Alternately, the nonlinear evolution produces a dominant wavelength that appears to be half the length of the analytically determined pattern. This may be attributed to the trend explained by Fig. 6, in which the nonlinear term possesses a wavelength twice that of the linearly stable pattern. The result from this simulation indicates that under evolution over long timeframes, the nonlinear term dominates the pattern selection. The final behavior of the distribution function, and the corresponding dominant wavelengths at long times, is identical to the results for all the 2-dimensional simulations at each respective angle of ion incidence.
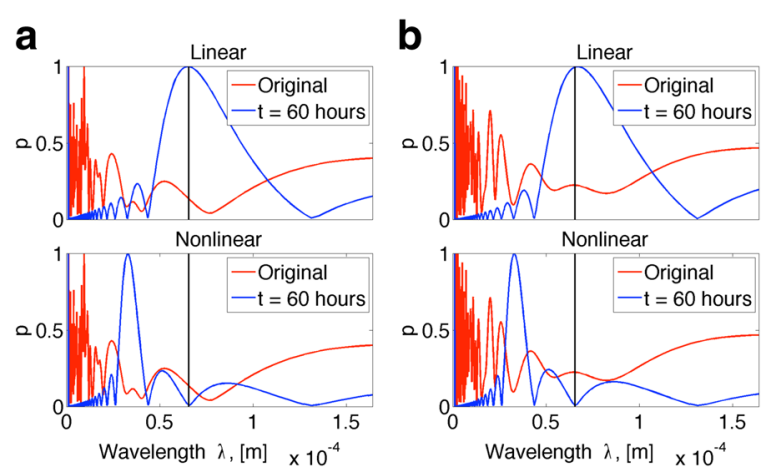

Fig. 9 Distribution function of the wavelengths corresponding to the surface modes present at time $t=0$ (red) and after $60 \mathrm{~h}$ of exposure (blue), for $\theta=0^{\circ}$. Note that in case of linear evolution (top), in both the (a) $x$-direction (b) $y$-direction, the dominant wavelength present in the spectrum matches closely with the analytical prediction according to linear stability analysis (shown by vertical black line). Nonlinear evolution (bottom) shows a final wavelength that is approximately half the analytical description, indicating the coupling of $2 \vec{q}$ has taken over the symmetry 
Additional simulations were performed at various angles of incidence to observe the effect of nonlinearities. The results of a simulation performed at $\theta=45^{\circ}$ are shown in Fig. 10, before and after $60 \mathrm{~h}$ of ion exposure. The linear results are similar to those at $\theta=0$, showing the coupled presence of all three wave vectors in the morphology. In the nonlinear case, contrasting surface morphologies can be seen for the respective angles of incidence. While an incidence angle $\theta=0^{\circ}$ yielded a clear dominant wavelength, simulations for $\theta=45^{\circ}$ showed the presence of coupling between differently oriented wave vectors, producing the dot pattern seen in Fig. 10c. At this angle, $\hat{v}_{x}=\hat{v}_{y}$, such that the $\mathrm{x}$ - and $\mathrm{y}$-components are equal in magnitude. In addition, there is orientational degeneracy of the dominant wavelength, however the same type of "dot" and ripple patterning is consistent across all simulations. This result further indicates competition between the wave vectors. The patterning in the nonlinear result for $\theta=45^{\circ}$ is unique in that the wavelengths controlling the patterns are again half the magnitude of the linear result. The conclusion that may be drawn for the nonlinear case is that in all directions, the wave vectors that emerge are twice in magnitude as in linear evolution. That is, the dominant wave vectors correspond to $2 \vec{q}_{1}, 2 \vec{q}_{2}$, and $2 \vec{q}_{3}$.

As noted previously, the orientation of the dominant wave vectors is determined by the relationship of the magnitudes of the second order curvature coefficients $\hat{v}_{x}$ and $\hat{v}_{y}$. This relationship is shown in Fig. 11, which displays the magnitudes of both terms over a range of angles of ion incidence, $\theta$. It can be seen that at $\theta=0^{\circ}$ and $\theta=45^{\circ}$, the magnitude of both values is equal, which explains why for both simulations the $\mathrm{x}$ - and $\mathrm{y}$-components of the ripple wave vectors were equal, and there was orientational degeneracy of the solution. It can also be seen than the magnitude of $\hat{v}_{x}$ is greater than $\hat{v}_{y}$ for all angles of incidence except between approximately $45^{\circ}$ and about $57^{\circ}$.

To observe the morphology under an angle of incidence in which $\hat{v}_{x}$ and $\hat{v}_{y}$ are not equal, simulations were performed on a randomized surface at $\theta=50^{\circ}$, where $\left|\hat{v}_{x}\right|<\left|\hat{v}_{y}\right|$. The results are shown in Fig. 12. It can be seen in Fig. 12b that the linear result looks similar to that of other angles of incidence, except that the orientation of the ripples is different due to the anisotropy of the second order terms. The nonlinear result (Fig. 12c) shows a
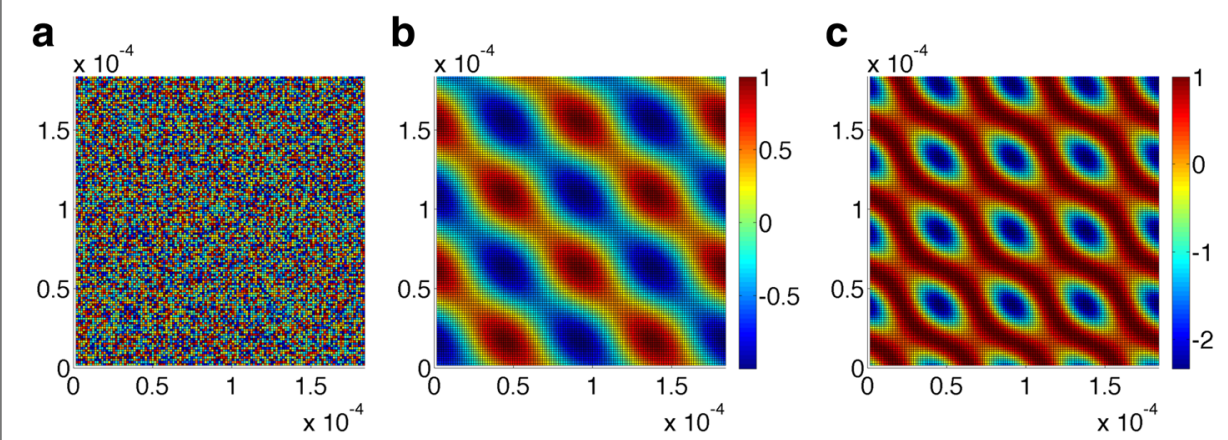

Fig. 10 Height data for the surface profiles of the (a) original surface prior to evolution, and surface after $60 \mathrm{~h}$ of evolution using (b) the linear evolution equation and (c) nonlinear evolution equation at $\theta=45^{\circ}$. The surface height is normalized to the maximum height, where the peaks are shown by the red regions while the troughs are shown as blue. The $x$ - and $y$-axes are expressed in [m] to show the feature size. The evolution parameters are as shown in Table 1. Both evolution regimes produce dots-on-ripples patterns, with contrasting characteristics 


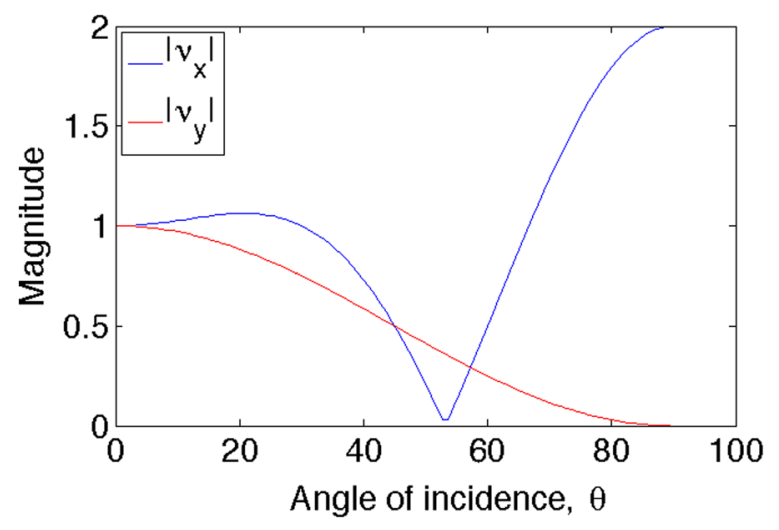

Fig. 11 Comparison of the second order curvature term coefficients magnitudes, $\left|\hat{v}_{x}\right|$ and $\left|\hat{v}_{y}\right|$. It can be seen the magnitude of $\hat{v}_{x}$ is greater than $\hat{v}_{y}$ for all angles except the range $45^{\circ}<\theta<\sim 57^{\circ}$

particularly unique pattern, where the multiples of diagonal wave vectors (i.e. $2 \vec{q}_{2}, 2 \vec{q}_{3}$ ) dominate the morphology and create the diamond patterns that are observed.

Figure 13 shows the convergence behavior of the evolution corresponding to Fig. 8. On the left is the linear evolution, where the dominant wavelength converges rather quickly, after $2.25 \mathrm{~h}$. The nonlinear evolution on the right side of the figure shows the solution initially settles near the analytical solution after $2.25 \mathrm{~h}$, but the final solution converges after $15.75 \mathrm{~h}$, where the dominant wavelength corresponds to half the wavelength of the analytical solution after the nonlinear term has taken over. Because of the random nature of the initial surface, the speed of convergence will vary for alternate simulations. Generally, the solution was found to converge at times under $10 \mathrm{~h}$ for the linear case, and $20 \mathrm{~h}$ for the nonlinear evolution. With this simulation, the displayed solution shows no variation for over $40 \mathrm{~h}$ of simulated exposure, indicating convergence.

\section{Conclusions}

This study highlights the pattern-forming characteristics of ion-bombarded surfaces according to a theoretical understanding of the physical processes. The outcomes have
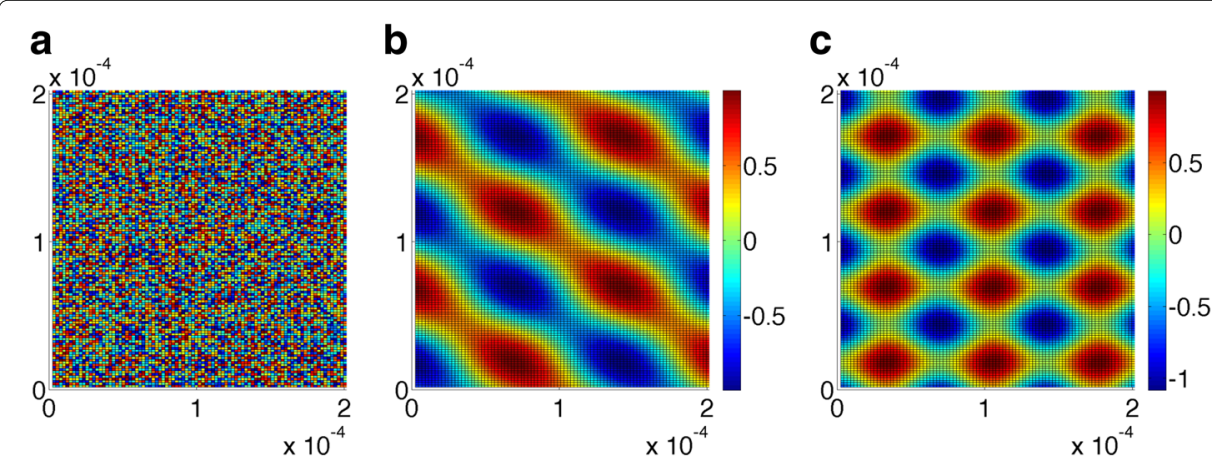

Fig. 12 Height data for the surface profiles of the (a) original surface prior to evolution, and surface after $60 \mathrm{~h}$ of evolution using (b) the linear evolution equation and (c) nonlinear evolution equation at $\theta=50^{\circ}$. The surface height is normalized to the maximum height, where the peaks are shown by the red regions while the troughs are shown as blue. The $x$ - and $y$-axes are expressed in [m] to show the feature size. The evolution parameters are as shown in Table 1 

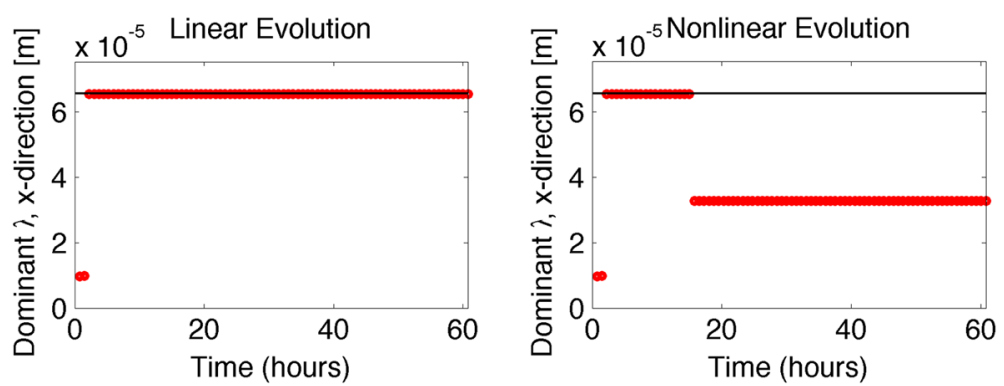

Fig. 13 Convergence of the dominant wavelength in the $x$-direction both linear evolution (left) and nonlinear evolution (right). Black horizontal line indicates analytical result. The nonlinear result shows that initially the morphology reaches a steady solution near the analytical result, but as time advances the nonlinear term takes over and the solution converges at this wavelength

been achieved through both an analytical examination of stability, as well as a computational investigation to provide insight where analytical theory is questionable. The comprehensive stability analysis provides a background for understanding the types of patterns that occur under various conditions relating to the evolution equation. Linear stability analysis provides the expected dominant wavelength magnitude of ripple formations, determined by the ion and material parameters. This furnishes a baseline to which the size of ripples may be compared, in order to track the presence of coupled modes resulting from the quadratic nonlinearity. The orientation of ripples is shown to be dependent on the most unstable wave vector. In addition, weakly nonlinear analysis demonstrated that the coupling of other modes can destabilize one-dimensional stripes in order to sustain hexagonal patterns. When the damping coefficient is set to zero $(\bar{\alpha}=0)$, the evolution operates outside the weakly nonlinear regime, such that the range of unstable wave vectors is maximized. Under such conditions, the evolution proceeds following the undamped KS equation form, presenting an area that has not been well investigated. In the undamped regime, the ripple orientation is not easily predicted and depends on the competition between modes. The selected mode for a particular initial surface configuration is controlled largely by the angle of ion incidence $\theta$, which controls the magnitude of the coefficients $\hat{v}_{x}$ and $\hat{v}_{y}$.

Computational simulations offer insight into the evolution behavior outside the weakly nonlinear regime, where the competition between wave vectors is not easily predicted. Therefore, the resulting patterns are dependent on the initial surface architecture, which influences the emergence of certain modes. For the 2-dimensional simulations, a randomized surface height profile has been selected in order to observe overall trends associated with the evolutionary mechanisms, rather than relying on the consistent influence of a particular surface configuration.

Simulations performed using the linear evolution equation have produced numerical results that demonstrated the formation of "dots-on-ripples" type patterning. The orientation of these ripples depends on the relationship of the curvature coefficients $\hat{v}_{x}$ and $\hat{v}_{y}$, where the components of the wave vectors are equal at angles where their magnitudes match (i.e. $\theta=0^{\circ}$ and $45^{\circ}$ ). Performing spectral analysis on the profiles show that the most dominant wavelength closely matches the analytical calculations for stable ripple formation. By introducing the nonlinear term to the evolution equation, it has been found that the effect of the nonlinearities is significant over long time spans, producing sharpened 
or enhanced symmetries. Spectral analysis as well as qualitative results have shown that at short times, the nonlinear term has little effect on the morphology of the surface profile. After a sufficient number of time steps, however, the nonlinear term grows rapidly and dominates the evolution equation. The nonlinear influence manifests itself by producing surface ripples with a dominant wavelength that is half the magnitude of what is expected according to linear stability. This result demonstrates the coupling of $2 \vec{q}_{c}$ as the fastest growing growing mode, following the effect of the quadratic nonlinearity. It is worth mentioning here that the nonlinearities in the evolution equation actually reflect the large local variations in the surface structure; an aspect that has not been considered in the original $\mathrm{BH}$ theory.

In the nonlinear case, the angle of ion incidence is found to be influential in the selected pattern formation. While normal incidence only resulted in dots-on-ripples patterning, $\theta=45^{\circ}$ resulted in the ripples becoming a clear dot pattern. That is, at $\theta=0^{\circ}$, the nonlinear mode oriented along $\vec{q}_{2}$ dominates the coupled modes of other orientations, while $\theta=45^{\circ}$ permits strong influence of multiple differently oriented modes. The nonlinear dots-on-ripples again reveal $2 \vec{q}_{c}$ to be the fastest growing mode, which contrasts with the linear result. The presence of nonlinearities has also been shown to require an extended speed of convergence compared with linear evolution, as the nonlinear term is initially not present in the symmetry, but eventually dominates the evolution.

\section{Acknowledgements}

This material is based upon work supported by the US Air Force Office of Scientific Research (AFOSR), under award number FA9550-16-1-0444.

\section{Authors' contributions}

CSRM and NMG prepared the manuscript and conceived the study. DW lead the nonlinear stability analysis and CSRM formulated the numerical simulations. All authors read and approved the final manuscript.

\section{Competing interests}

The authors declare that they have no competing interests.

\section{Publisher's Note}

Springer Nature remains neutral with regard to jurisdictional claims in published maps and institutional affiliations.

Received: 9 March 2017 Accepted: 9 May 2017

Published online: 21 June 2017

\section{References}

MA Makeev, R Cuerno, A-L Barabasi, Morphology of ion-sputtered surfaces. Nucl. Inst. Methods Phys. Res. Sect. B: Beam Interactions Mater. Atoms. 197(3), 185-227 (2002)

G Costantini, S Rusponi, FB de Mongeot, C Boragno, U Valbusa, Periodic structures induced by normal-incidence sputtering on ag (110) and ag (001): flux and temperature dependence. J. Phys. Condens. Matter. 13(26), 5875 (2001)

S Habenicht, Morphology of graphite surfaces after ion-beam erosion. Phys. Rev. B. 63(12), 125419 (2001)

M Navez, C Sella, D Chaperot, Nonlinear ripple dynamics on amorphous surfaces patterned by ion beam sputtering Czetch Repub. Acad. Sci. 254, 240 (1962)

S Rusponi, C Boragno, U Valbusa, Ripple structure on ag (110) surface induced by ion sputtering. Phys. Rev. Lett. 78(14), 2795 (1997)

U Valbusa, C Boragno, FB de Mongeot, Nanostructuring surfaces by ion sputtering. J. Phys. Condens. Matter. 14(35), 8153 (2002)

P Sigmund, A mechanism of surface micro-roughening by ion bombardment. J. Mater. Sci. 8(11), 1545-1553 (1973)

RM Bradley, JME Harper, Theory of ripple topography induced by ion bombardment. J. Vac. Sci. Technol. A. Vac. Surf. Films. 6(4), 2390-2395 (1988)

D Walgraef, NM Ghoniem, J Lauzeral, Deformation patterns in thin films under uniform laser irradiation. Phys. Rev. B. 56(23), 15361 (1997)

J Lauzeral, D Walgraef, NM Ghoniem, Rose deformation patterns in thin films irradiated by focused laser beams. Phys. Rev. Lett. 79(14), 2706 (1997)

J Muñoz-García, L Vazquez, M Castro, R Gago, A Redondo-Cubero, A Moreno-Barrado, R Cuerno, Self-organized nanopatterning of silicon surfaces by ion beam sputtering. Mater. Sci. Eng. R: Rep. 86(1-44) (2014)

P Sigmund, Theory of sputtering. i. sputtering yield of amorphous and polycrystalline targets. Phys. Rev. 184(2), 383 (1969)

R Cuerno, A-L Barabási, Dynamic scaling of ion-sputtered surfaces. Phys. Rev. Lett. 74(23), 4746 (1995) 
WW Mullins, Theory of thermal grooving. J. Appl. Phys. 28(3), 333-339 (1957)

RJ Asaro, WA Tiller, Interface morphology development during stress corrosion cracking: Part i.via surface diffusion. Metall. Trans. 3(7), 1789-1796 (1972)

WH Yang, DJ Srolovitz, Surface morphology evolution in stressed solids: surface diffusion controlled crack initiation. J. Mech. Phys. Solids. 42(10), 1551-1574 (1994)

C Godrèche, Solids far from Equilibrium, volume 1. (Cambridge University Press, Cambridge, 1991)

SF Edwards, DR Wilkinson, The surface statistics of a granular aggregate. Proc. R. Soc. Lond. A Math. Phys. Eng. Sci. 381(1780), 17-31 (1982)

J Villain, Continuum models of crystal growth from atomic beams with and without desorption. J. Phys. I. 1(1), 19-42 (1991)

Y Kuramoto, T Tsuzuki, Persistent propagation of concentration waves in dissipative media far from thermal equilibrium. Prog. Theor. Phys. 55(2), 356-369 (1976)

Gl Sivashinsky, Nonlinear analysis of hydrodynamic instability in laminar flames: Derivation of basic equations. Acta Astronaut. 4(11), 1177-1206 (1977)

A Keller, S Facsko, lon-induced nanoscale ripple patterns on si surfaces: theory and experiment. Materials. 3(10), $4811-4841$ (2010)

S Facsko, T Bobek, A Stahl, H Kurz, T Dekorsy, Dissipative continuum model for self-organized pattern formation during ion-beam erosion. Phys. Rev. B. 69(15), 153412 (2004)

RM Bradley, Redeposition of sputtered material is a nonlinear effect. Phys. Rev. B. 83(7), 075404 (2011)

KH De Grys, A Mathers, B Welander, V Khayms, Demonstration of 10,400 hours of operation on 4.5 kw qualification model hall thruster. AIAA Pap, 6698 (2010)

N Ghoniem, D Walgraef, Instabilities and Self-organization in Materials. (Oxford Univ. Press, Oxford, 2008)

D Walgraef, Spatio-Temporal Pattern Formation: with Examples from Physics, Chemistry, and Materials Science. (Springer Verlag, Berlin, 1997)

FC Motta, PD Shipman, RM Bradley, Highly ordered nanoscale surface ripples produced by ion bombardment of binary compounds. J. Phys. D. Appl. Phys. 45(12), 122001 (2012)

FC Motta, PD Shipman, RM Bradley, Theory of nanoscale pattern formation produced by oblique-incidence ion bombardment of binary compounds. Phys. Rev. B. 90(8), 085428 (2014)

G Dewel, S Métens, M'F Hilali, P Borckmans, CB Price, Resonant patterns through coupling with a zero mode. Phys. Rev. Lett. 74, 4647 (1995)

SM Cox, PC Matthews, Instability and localisation of patterns due to a conserved quantity. Phys. D. 175, 196-219 (2003)

JM Hyman, B Nicolaenko, The Kuramoto-Sivashinsky equation: A bridge between pdes and dynamical systems. Phys. D. 18, 113-126 (1986)

JM Hyman, B Nicolaenko, S Zaleski, Order and complexity in the kuramoto-sivashinsky model of weakly turbulent interfaces. Phys. D. 23, 265-292 (1986)

IG Kevrekidis, B Nicolaenko, JC Scovel, Back in the saddle again: A computer assisted study of the kuramoto-sivashinsky equation. SIAM J. Appl. Math. 50, 760-790 (1990)

P Cvitanovíc, RL Davidchack, E Siminos, On the state space geometry of the kuramotosivashinsky flow in a periodic domain. SIAM J. Appl. Dyn. Syst. 9(1), 1-33 (2010)

M Paniconi, KR Elder, Stationary, dynamical, and chaotic states of the two-dimensional damped kuramoto-sivashinsky equation. Phys. Rev. E. 56(3), 2713-2721 (1997)

H Gomez, J Paris, Numerical simulation of asymptotic states of the damped kuramoto-sivashinsky equation. Phys. Rev. E. 83(4), 046702 (2011)

M Rost, J Krug, Anisotropic kuramoto-sivashinsky equation for surface growth and erosion. Phys. Rev. Lett. 75, 3894-3898 (1995)

E Vitral, Nano-patterning of surfaces by ion sputtering: Numerical study of the anisotropic damped kuramoto-sivashinsky equation. Master's thesis, Universidade do Estado do Rio de Janeiro, (2015)

C Misbah, A Valance, Secondary instabilities in the stabilized kuramoto-sivashinsky equation. Phys. Rev. E. 49(1), 166-183 (1994)

CSR Matthes, NM Ghoniem, GZ Li, TS Matlock, DM Goebel, CA Dodson, RE Wirz, Fluence-dependent sputtering yield of micro-architectured materials. Appl. Surf. Sci. 407C, 223-235 (2017)

GZ Li, TS Matlock, DM Goebel, RE Wirz, CSR Matthes, NM Ghoniem, in In situ plasma sputtering and angular distribution measurements for structured molybdenum surfaces. Plasma Sources Science and Technology 26. vol. 6, (2017), p. 065002 